\title{
Integrierte Mikrosysteme für die Gas- und Flüssigkeitsanalytik
}

\author{
Prof. Dr.-Ing. habil Jörg Müller, Institut für Mikrosystemtechnik, \\ TU Hamburg-Harburg, Eißendorferstr. 42, 21073 Hamburg, j.mueller@tuhh.de
}

\section{Zusammenfassung}

Komplexe physikalische Analysesysteme lassen sich mit Hilfe der Mikrosystemtechnik reproduzierbar und preisgünstig miniaturisieren, ohne dass darunter die Systemeigenschaften gegenüber makroskopischen Systemen maßgeblich eingeschränkt werden. Allerdings erfordern sie i.a. die Nutzung alternativer physikalischer Prinzipien in der Analytik und auch der Elektronik in Hard- und Software. Als Beispiele für Mikroanalysesysteme werden der Gaschromatograph, das Massenspektrometer, der Flammenionisationsdetektor und der paramagnetische Sauerstoffsensor sowie periphere integrierbare Subsysteme wie Pumpen und Druckminderer vorgestellt.

\section{Einführung}

In vielen Bereichen der Grundlagenforschung, der Technik, Medizin und Umweltmesstechnik sind zur Analyse von Stoffsystemen physikalische Messverfahren etabliert. Gegenüber der Alternative, chemischen Wechselwirkungen zu nutzen, sind sie besonders selektiv, empfindlich und langzeitstabil. Geräte wie optische (FTIR) und Massenspektrometer (MS), Flüssigkeits- (HPLC) und Gaschromatographen (GC). Flammenionsationsdetektoren (FID) oder auch paramagnetische Sauerstoffsensoren sind das Rückgrat dieser Analytik. Allerdings ist die Mehrzahl der gegenwärtig verfügbaren Analysesysteme für den Laboreinsatz ausgelegt, da sie bezüglich Gewicht, Volumen, Medienbedarf, thermischen und mechanischen Randbedingungen, insbesondere aber auch Kosten für Beschaffung und Betrieb anspruchsvoll sind. Die Option, derartig komplexe Messgeräte zu miniaturisieren, um so portable, medienschonende und dabei preisgünstige und leicht zu bedienende Systeme zu entwickeln, bietet grundsätzlich die Mikrosystemtechnik. Dazu gibt es weltweit vielfache Anstrengungen, durchgängige Systemlösungen sind bisher aber sehr selten. Als wesentliches Hindernis erweist sich die Notwendigkeit, dass die Entwicklung eines vollständigen Analysesystems in allen Subsystemen und Komponenten aufeinander abgestimmt sein muss. Darüber hinaus sind vielfach gegenüber Makrosystemen alternative, spezifisch auf die Möglichkeiten aber auch Einschränkungen der Mikrosystemtechnik abgestimmte physikalische Messprinzipien zu verwenden, die vielfach völlig neuartige bzw. darauf adaptierte Lösungen erfordern. Dies gilt gleichermaßen für die Elektronik in Hard- und Software, bei der wegen der kleinen Dimensionen z.B. niedrige Spannungen bei hohen Frequenzen und sehr niedrige Rauschpegel z.B. zur Messung sehr kleiner Ströme erforderlich und Schaltungen für neue Messprinzipien zu entwickeln sind.

Mit dem häufig gewählten Weg, Teilsysteme in Mikrotechnik in etablierte Standardsysteme zu implementieren, werden nur selten der Vorteile dieser Technologie vollständig genutzt.

Im Folgenden werden einige solcher hochintegrierten Systeme vorgestellt werden, deren Grundlagen jeweils zunächst an der TUHH untersucht wurden und die dann z.T. zusammen mit Industriepartnern zu Demonstratoren bis hin zu Produkten entwickelt worden sind. Einige haben sie sich schon im industriellen Umfeld bewährt, andere sind auf dem Wege zu technischen Produkten. 


\section{Die Technologie}

Diese Systeme werden als Glas-Silizium-Glas-Sandwich im Batchprozess (typisch 10 bis 100 Systeme auf 100mm-Substrat) mit industriell eingeführten Prozessen hergestellt. Die Silizium-Ebene enthält die komplexen Strukturen des Mikrosystems, vorzugsweise erzeugt mit anisotropem Hochra-

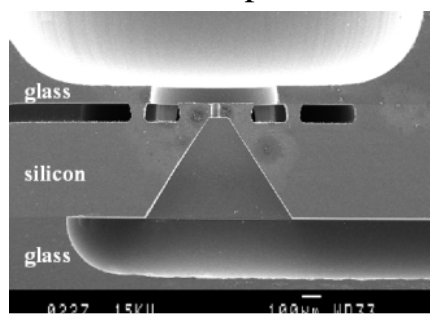

Bild 1: Aufbau eines GlasSilizium-Glas-Mikrosystems ten-Plasmaätzen (DRIE), das die Herstellung 2 1/2-dimensionaler Strukturen erlaubt. Im Glassubstrat werden nasschemisch Kanalstrukturen und Durchbrüche für die Medienzufuhr geätzt. Metallstrukturen für Leiterbahnen und - vorwiegend freitragend - für integrierte Sensoren für Fluss-, Wärmeleitungs- oder Drucksensoren sowie Elektroden zur Strommessung werden je nach Anforderung in der Siliziumebene oder den Glasebenen mit Hilfe von Dünnschicht- und Photoätzprozessen realisiert. Das REM-Bild (Bild 1) zeigt einen Ausschnitt aus einer solchen Struktur.

Diese einheitliche Technologie und die typischen Systemflächen von $1 \mathrm{~cm}^{2}$ gewährleisten die Kombinierbarkeit unterschiedlicher Systeme und senken die Entwicklungs- und - in Verbindung mit der parallelen Bearbeitung im Batch - die Produktionskosten. Über den Aufbau der Systeme inklusive der Elektronik hinaus ist i.a. auch notwendig, die Peripherie wie die Medienver- und -entsorgung, die Probenzufuhr und die Aufbau-und Verbindungstechnik insbesondere im Hinblick auf minimale Totvolumina an solch kleine Systeme zu adaptieren.

\section{Mikroanalysesysteme}

Aus der Fülle möglicher Realisierungen sollen hier einige Systeme beschrieben werden

\section{Der Mikro-Gaschromatograph (GC)}

Einen vollständigen Mikro-GC zeigt Bild 2. Er nimmt einschließlich Medienversorgung und Auswer-

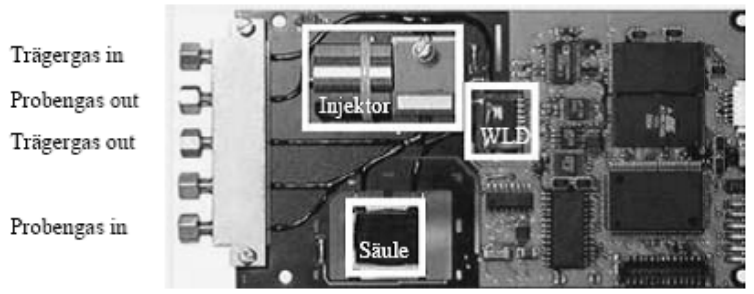

Bild 2: Mikrogaschromatograph mit Auswerteelektronik (SLS-Microtechnology) teelektronik die Fläche von 129x65 $\mathrm{mm}^{2}$ ein (SLS-Microtechnology). Auf einer Fläche von etwa $20 \times 25 \mathrm{~mm}^{2}$ ist in einem Silizium-GlasVerbund eine zylindrische, ca. $90 \mathrm{~cm}$ lange, als Mäander ausgeführte Trennsäule integriert. Dazu werden jeweils halbkreisförmige Querschnitte isotrop in die Substrate geätzt, in einem Plasmapolymerisationsprozess mit einer Silikon-ähnlichen stationären Phase ausgekleidet und durch anodisches Bonden verbunden. Alternativ werden auch gestopfte Säulen durch das Auffüllen der Kanäle mit Adsorbenzien realisiert. Zur Kontrolle des Gasflusses sind in den Kanal zusätzlich Anemometerstrukturen in der Form freitragender Dünnschicht-Platinwendel integriert (s.u.). Sie sind in Brückenschaltung aufgebaut und können so den Einflusses von Gasart, Druck und Temperatur unterdrücken. Aufgebracht auf einer Keramikheizplatte kann die Säule wegen der kleinen thermischen Masse und hohen Wärmeleitung von Keramik und Silizium sowie der hohen Temperaturbeständigkeit der plasmapolymerisierten stationären Phase innerhalb von jeweils ca. 60 Sekunden von Raumtemperatur auf bis $\mathrm{zu}>300^{\circ} \mathrm{C}$ aufheizt und durch einen Lüfter schnell wieder abkühlt werden. Dies ermöglicht es - im Gegensatz zu üblichen GC-Anordnungen mit KapillarsSäulen - durch thermisches Zykeln die Trennung zu dynamisieren und damit die Messzeiten signifikant zu verkürzen. Darüber hinaus kann die Reinigung der Säule durch kurzzeitiges Ausheizen bei hoher Temperatur erfolgen und damit 
ein sonst übliches Spülen oder gar Rückspülen vermieden werden, was signifikant die Messabfolge beschleunigt und den Peripherieaufwand reduziert. So können z.B. Chromatogramme von Kohlenwasserstoffgemischen (z.B. C1 bis C10) ca. alle $2 \mathrm{~min}$ aufgenommen werden. Das System benötigt ca. $100 \mu \mathrm{l} / \mathrm{min}$ für die mobile Phase $(\mathrm{He})$ und das injizierte Probenvolumen liegt bei wenigen $100 \mathrm{nl}$. Die Konzentrationen der in der Säule getrennten Gas komponenten werden mit einem hochempfindlichen Wärmeleitungsdetektor in einer dem

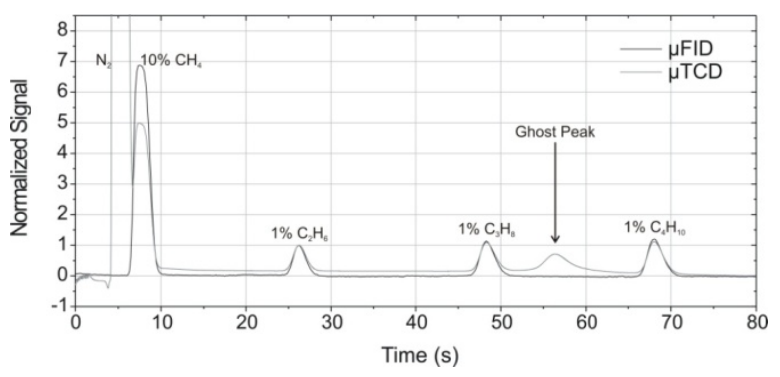

Bild 3: $\mu$-GC-Chromatogramm eines Kohlenwasserstoffgasgemischs ( $\mathrm{N} 21$ ) gemessen mit $\mu$-WLD und $\mu$-FID Heizdrahtanemometer ähnlichen, auf konstante Temperatur geheizten Struktur in Brückenanordnung bis zu Konzentrationen von wenigen 10ppm bestimmt. Bild 3 zeigt ein solches Chromatogramm.

\section{Massenspektrometer}

Eine sehr viel komplexere Struktur ist das planar-integrierte Massenspektrometer (PIMMS) (Bild 4).
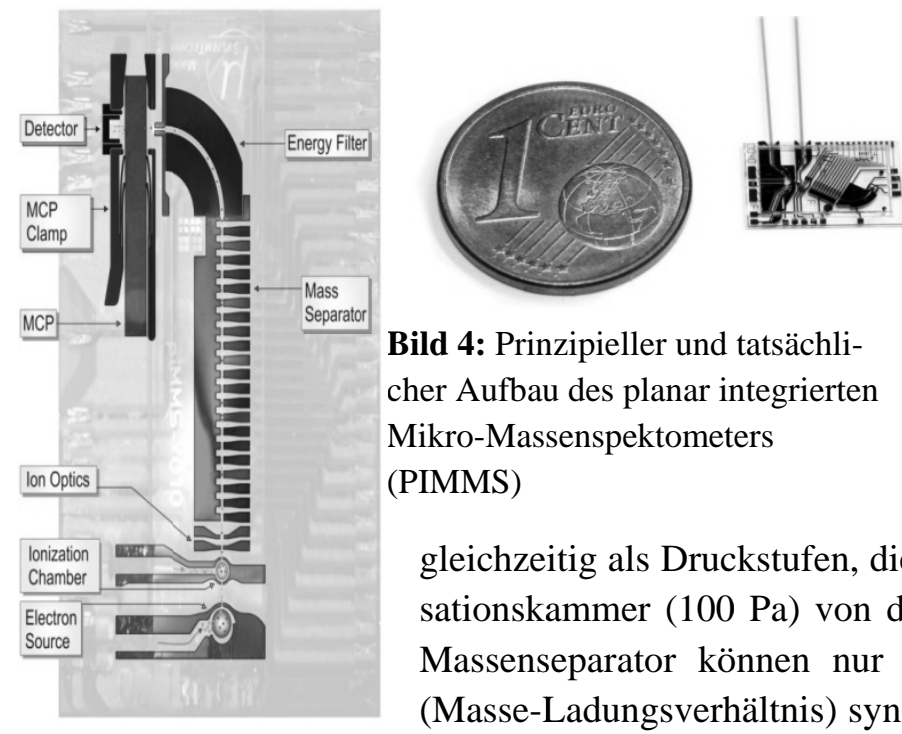

Bild 4: Prinzipieller und tatsächlicher Aufbau des planar integrierten Mikro-Massenspektometers (PIMMS) gleichzeitig als Druckstufen, die die relativ hohen Drücke in Plasma und Ionisationskammer (100 Pa) von den niedrigen außerhalb (0,1 Pa) trennen. Den Massenseparator können nur die Ionen passieren, deren Geschwindigkeit (Masse-Ladungsverhältnis) synchron mit einer im Wanderfeld erzeugten feldfreien Zone den Elektrodenkamm durchlaufen. Zur Gewährleistung einer hohen Massenauflösung folgt ein als Kreissegment ausgeführter Energiefilter. Der Strom wird detektiert mit einem Faradaydetektor, ggf. angeordnet hinter einer in einer selbstjustierenden und kontaktierenden Federanordnung hybrid integrierten Mikrokanalplatte (MCP), die den Ionenstrom durch Sekundärelektronenvervielfachung verstärkt. Die über die Steuerelektronik automatisch geregelten Systemspannungen liegen bei einigen $10 \mathrm{~V}$ bis zu $150 \mathrm{~V}$ für die Elektronenund Ionenoptiken und bei ca. $1 \mathrm{kV}$ für die MCP. Das Wanderfeld basiert auf 5V-Rechtecksignalen zur Massenauswahl, gescannt zwischen 1 und $270 \mathrm{MHz}$ bei Anstiegszeiten $<1 \mathrm{~ns}$. Das Gesamtsystem ist $7 \times 12 \mathrm{~mm}^{2}$ klein und wird auf $<10^{-1} \mathrm{~Pa}$ evakuiert. Der Gasverbrauch für den Betrieb das Plasmaquelle (Ar) liegt wie für das Probengas bei

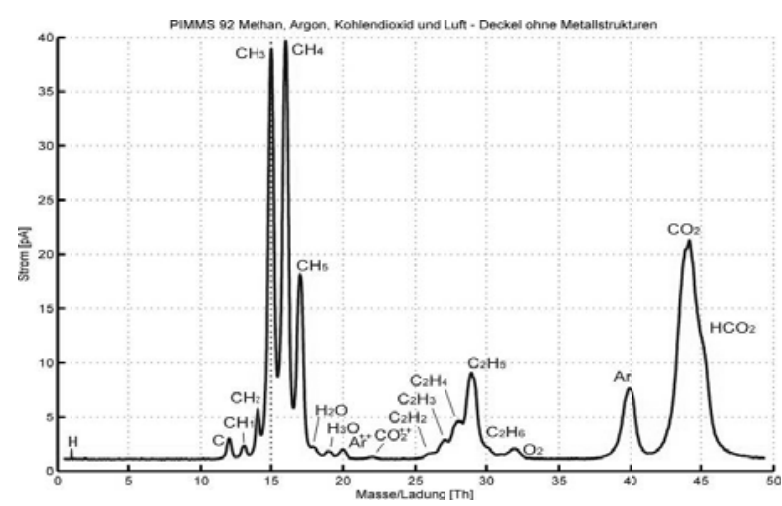

Bild 5: Methanspektrum, gemessen mit einem PIMMS 
$100 \mu \mathrm{l} / \mathrm{min}$. Bild 5 zeigt ein Massenspektrum von Methan, die Massenauflösung liegt gegenwärtig bei $>$ 40, die Empfindlichkeit bei <100ppm. Die Spektren werden vollautomatisch aufgenommen mit einer spezifisch für dieses System entwickelten Hard- und Software. Mit den erforderlichen Probenvolumina von ca. $100 \mu \mathrm{l} / \mathrm{min}$ kann es sehr gut mit dem o.g. $\mu \mathrm{GC}$ kombiniert werden (GC-MS).

\section{Flammenionisationsdetektor (FID)}

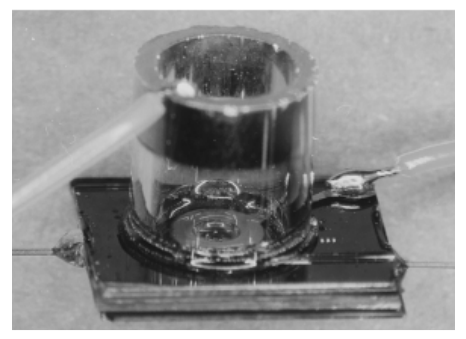

Bild 6: Hybrider MikroFlammenionisationsdetektor

Als besonders empfindlicher Detektor für Kohlenwasserstoffe wird alternativ zum Wärmeleitungsdetektor oder Massenspektrometer besonders in Kombination mit Gaschromatographen der Flammenionisationsdetektor (FID) mit einer Knallgasflamme $\left(\mathrm{H}_{2}, \mathrm{O}_{2}\right)$ verwendet. Er lässt sich sehr vorteilhaft in Mikrotechnik realisieren, da seine wesentlichen Strukturen eine Düsenanordnung zur Erzeugung und Formung der Flamme und Injektion des Probengases und Kanalstrukturen zur Zufuhr von Brenn- und Probengasen sind. Eine Elektrodenanordnung fängt die in der Flamme entstehenden Ionen als Maß für die Kohlenstoffkonzentration auf. Wegen der in Mikrosystemtechnik reproduzierbar realisierbaren kleinen Düsen-

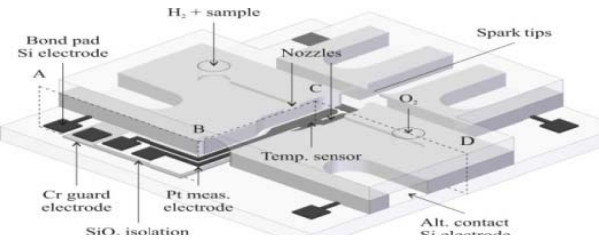

Bild 7: Planarer Aufbau des $\mu$-FIDs

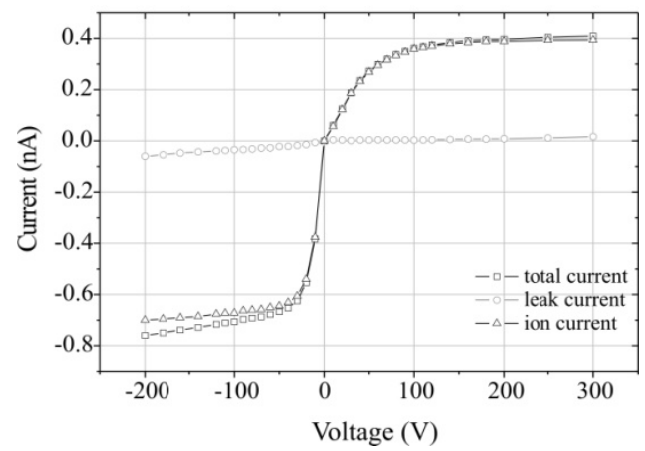

Bild 8: Strom-Spannungs-Charakteristik eines $\mu$ FID strukturen kann prinzipiell der Nachteil relativ hoher Gasverbräuche (insbesondere Wasserstoff) vermieden werden, die seine Verbreitung wegen der Sicherheitsauflagen insbesondere außerhalb von Laboranwendungen erheblich beeinträchtigen. Eine erste Realisierung eines solchen Systems als Mikrosystem zeigt Bild 6. Es weist den gewünschten geringen Brenngasverbrauch (35 ml/min) bei hoher Empfindlichkeit $(<1 \mathrm{ppm})$ auf, allerdings ist es nicht vollständig integriert, da die Messelektrode als metallisierter Quarz-Zylinder aufgesetzt ist. Dadurch ist auch die Abschirmung der Umgebung von der Flamme nicht ausreichend. Den Aufbau eines vollständig planar integrierten und damit von der Außenwelt abgeschirmten FID zeigt Bild 7. Hier liegen Düsen und Flamme in der Systemebene (Silizium), und die Flamme ist zwischen zwei Glasplatten eingeschlossen, was auch den Energieverlust und damit die notwendige Brenngasmenge weiter verringert $(<20 \mathrm{ml} / \mathrm{min})$. Dieses System ist außerdem nach dem Gegenstromprinzip aufgebaut, d.h. Sauerstoff und mit der Probe vermischter Wasserstoff werden von gegenüberliegenden Seiten der Flamme zugeführt. Dies ermöglicht eine örtlich und zeitlich sehr stabile Flammenfront. Darin verweilt die Probe lange und wird sehr effektiv ionisiert. Zusammen mit den kurzen Abständen zu den in der Kammer integrierten Ionenfangelektroden werden schon bei niedrigen Spannungen (ca. $10 \mathrm{~V}$ gegenüber 150 bis $500 \mathrm{~V}$, Bild 8) alle Ionen eingefangen. Da die Flamme bis auf kleine Auslassöffnungen rundum von der Silizium-Glas-Struktur umschlossen ist, ist keine Stützluft erforderlich und die thermische Abschirmung ermöglicht den Betrieb mit $\mathrm{H}_{2}$ - bzw. $\mathrm{O}_{2}$-Flüssen von ca. $10 \mathrm{ml} / \mathrm{min}$, Mengen, die statt aus Gasflaschen (Sicherheitsauflagen) auch durch Elektrolyse von Wasser bereitgestellt werden können. Die Systemempfindlichkeit entspricht der kommerzieller makroskopischer Systeme ( $<1 \mathrm{ppm})$. Das Chromatogramm des Mikro-GC von Bild 3 zeigt auch die Antwort eines nachgeschalteten FIDs, der, da er nur Kohlenwasserstoffverbindungen registriert, den vom WLD detektierten unerwünschten Wasserpeak nicht enthält. 


\section{Paramagnetischer Sauerstoffsensor}

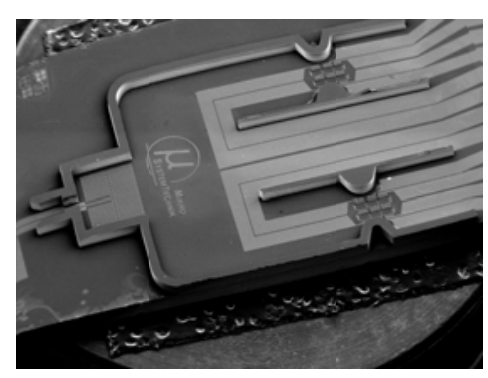

Bild 9: Ausschnitt des paramagnetischen Sauerstoffsensors mit Anemometern in Referenzkanal (oben) und Messkanal und Magneten (unten)
Die Mikrotechnik erlaubt auch, in der Makrowelt z.B. aufgrund von Fertigungstoleranzen oder minimalen Dimensionen nicht nutzbare Messprinzipien einzusetzen. Ein Beispiel ist der paramagnetische Sauerstoffsensor nach Bild 9. Durch eine parallele Anordnung von sehr genau definierten Kanälen mit integrierten Heizdrahtanemomentern - einem Hauptkanal, einem Messkanal rechts, ein Referenzkanal links - sowie einem seitlich angeordneten Magneten wird die Sauerstoffkonzentration durch die Ablenkung des paramagnetischen Gases aus dem Hauptkanal in den Messkanal, bei gleichzeitiger Verringerung des Flusses im Referenzkanal, bestimmt. Bild 10 zeigt die simulierten Flüsse durch ein solches System bei reinem Stickstoff- bzw. reinem Sauerstoffstrom.

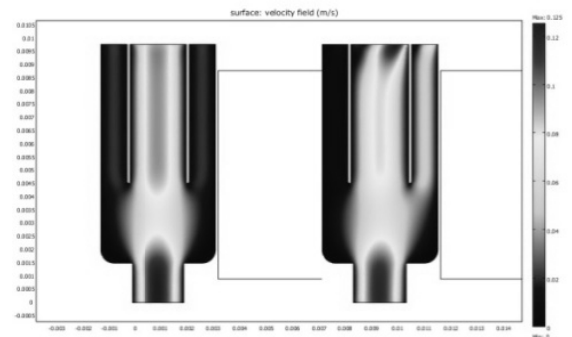

Bild 10: Simulierte Flüsse für reinen Stickstoff- bzw. Sauerstofffluss

\section{Periphere Komponenten.}

Wie schon o.g. werden zum Betrieb solcher Mikroanalysesysteme zusätzlich periphere Komponenten wie Massenflussmesser, Druckmesssysteme, Druckstufen oder Verdampfer benötigt, aber auch Pumpen und Ventile. Beispielhaft sollen einige solcher, vielfach in die beschriebenen Systeme integrierter bzw. integrierbarer Komponenten aufgeführt werden:

\section{Heizdraht-Anemometer, Wärmeleitungsdetektor und Pirani-Druckmesser}

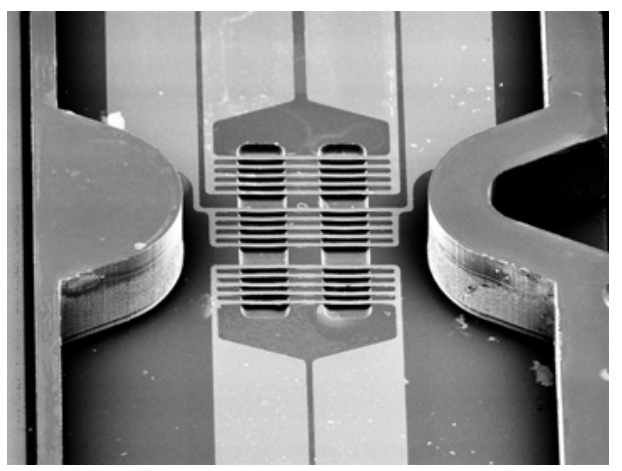

Diese für maximale Wechselwirkung mit dem Medium und geringster Wärmekapazität als mittig im jeweiligen Medientransportkanal oder mit definiertem Abstand zur Wand freitragend angeordneten elektrisch beheizten Widerstandstrukturen, optimal ausgeführt als Mäander, sind bevorzugt als Platin-Dünnschichten realisiert, um die erforderliche hohe thermische, vor allem aber chemische Stabilität zu ermöglichen. Als Anemometer werden drei in Reihe geschaltete Strukturen, mit einer elektrisch geheizten Struktur in der Mitte betrieben (Bild 12), ggf. auch in einer Halbbrücke verschaltet, wenn sie z.B. im paramagnetischen Sauerstoffsensor zur Messung von Differenzflüssen eingesetzt werden.

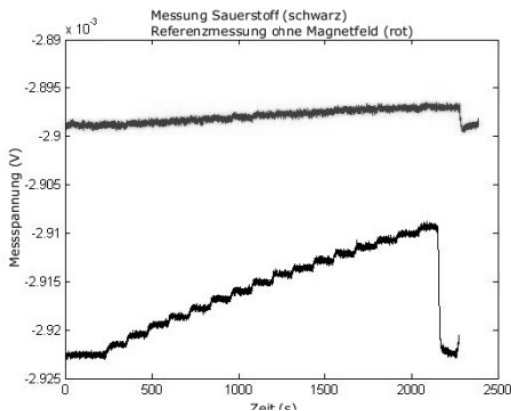

Wird die Sauerstoffkon$\left(\mathrm{N}_{2}, \mathrm{O}_{2}, 10 \mathrm{ml} / \mathrm{min}\right)$ in 5\%-Stufen erhöht, ergibt gestellte Verlauf (unbearbeitete Rohdaten).

Bild 11: Messung eines Sauerstoff-Stickstoff-Gemischs bei Variation der Sauerstoffkonzentration in 5\%-Schritten mit (unten) und ohne Magnetfeld (oben) 
Bei Wärmeleitungsdetektoren und Druckmessern auf Basis des Pirani-Effekts werden bevorzugt vier solcher Strukturen in einer Wheatstone`schen Brücke so verschaltet, dass zwei im Messkanal, möglichst in einem strömungsfreien Bereich zur Unterdrückung der Konvektion und zwei in Kammern mit in Art und Druck konstantem Medium eingeschlossen sind. Während der WLD optimal in Kanalmitte angeordnet ist, muss die freitragende Leiterbahn beim Pirani-System in einem Abstand, der geringer ist als die jeweilige freie Wegelänge von einer gut wärmeableitenden Wand angeordnet sein. Dies sind bei $10^{5}$ Pa wenige $100 \mathrm{~nm}$, bei 1 Pa schon mehrere $100 \mu \mathrm{m}$. Durch die kleinen Strukturen und ihre gute Ankopplung sind mit solchen WLDs Empfindlichkeiten im ppb-Bereich, für die PiraniDruckmesssysteme Messbereiche von jeweils 2-3 Größenordnungen und mit gestuften Abständen ein Intervall von $10^{-1}$ bis $10^{6} \mathrm{~Pa}$ integriert erreichbar. Bild 13 und Bild 15 zeigen eine solche Strukturen und typische Charakteristiken.

\section{Verdampfer und Druckstufen}

Die hochgenaue Strukturierung von Kanälen ggf. in Kombination

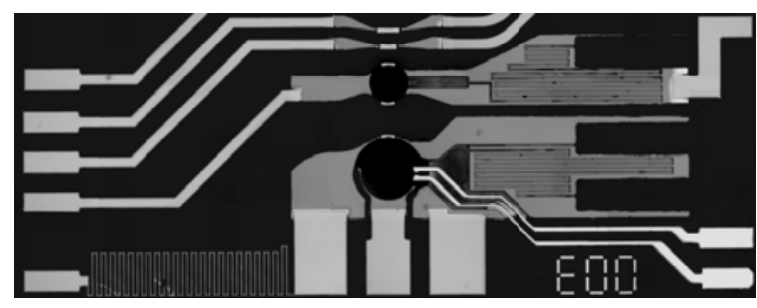

Bild 14: Druckminderungsstufe in PIMMS integriert

mit lokalen Verteilstrukturen ermöglicht einerseits definierte Druckreduktionen bei bekanntem Probenfluss für

Messsystem, die bei deutlich niedrigerem Druck arbeiten als der der Medienversorgung bzw. des Analyten durch Integration definierter Flusswiderstände auch als Kaskaden (Bild 14). Wegen der

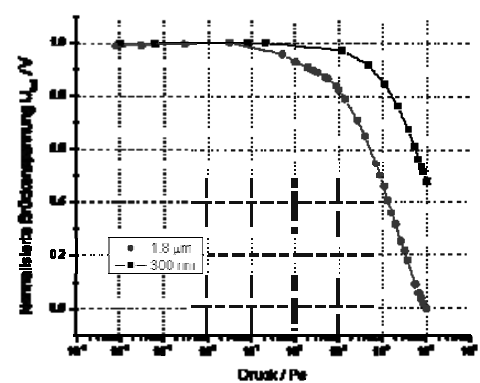

Bild 13: Charakteristik von Piranielementen mit unterschiedlichem Abstand zur Wand hohen Wärmeleitfähigkeit und geringen Wärmekapazität können solche Kanäle dauerhaft beheizt mit geringem Energieaufwand als Flüssigkeitsverdampfer bevorzugt in Kombination mit einer solchen Druckstufe eingesetzt werden. Alternativ lassen sich vorzugsweise dynamisch arbeitende Verdampfer zur - auch örtlich und zeitlich - gezielten Probennahme realisieren, die Dampfstöße durch Laserpulse erzeugen, die die hohe Absorption und geringe Wärmekapazität von Silizium zur Verdampfung und die hohe Transparenz des Deckglases zur verlustarmen Transmission nutzen.

\section{Pumpen}

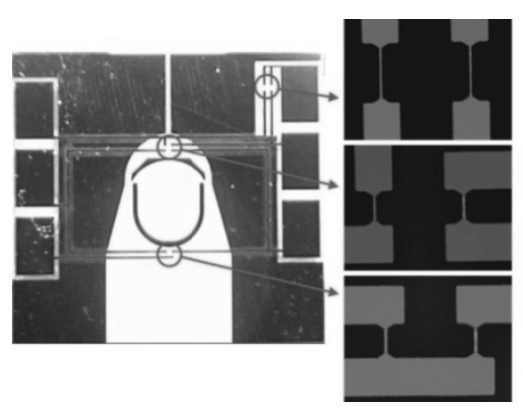

Bild 15: Planare Diffusionspumpe in Mikrosystemtechnik mit integrierten Pirani-Messystemen
Pumpen zum Transport von Gasen und Flüssigkeiten in Mikrosystemtechnik sind seit vielen Jahren bekannt und eingesetzt. Sie werden auch mit unterschiedlichen Aktivierungsprinzipien betrieben. Bisher nicht verfügbar sind Vakuumpumpen in dieser Technologie, so das Mikroanalysesysteme, die geringe Gasdrücke benötigen, die

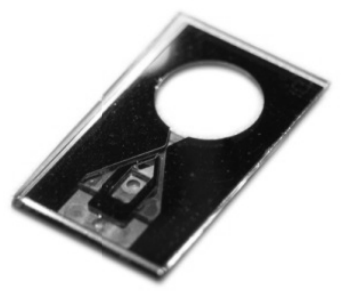

Bild 16: Düsenanordnung für Strahldüse
z.B. das Massenspektrometer, noch mit großvolumigen Pumpen 


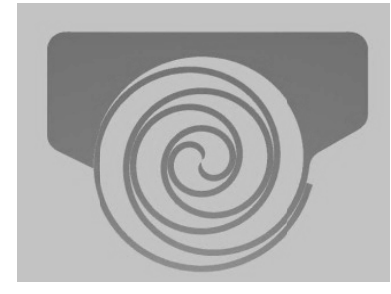

Bild 17: Prinzip der Scrollpumpe

betrieben werden müssen. Unterschiedliche Ansätze und erste Ergebnisse zu Pumpenstrukturen in Mikrosystemtechnik, die auf Prinzipien wie der Diffusion (Bild 15) oder der Mitnahme im Gasstrahl (Bild 16), der Verdrängung (Bild 17) oder der Adsorption (Bild 18) beruhen, sind möglich. Während Verdrängerpumpen wegen der kleinen Volumina und geringen Kompressionsverhältnis in Mikrosystemtechnik begrenzte Pumpleistungen und Druckminderungen erwarten lassen, sind mit Diffusions- und Strahlpumpen hohe Kompressionen und damit geringe Drücke prinzipiell möglich, wenn eine effektive Pumpmedienrückführung durch Kombination von Kondensierung und Flüssigkeitsrückführung über Kapillarstrukturen als Druckstufe gelingt. Bei Absorberpumpen, die vorzugsweise im Wechselbetrieb arbeiten sollten, lassen sich z.B. durch Einsatz von Peltierelementen zur Kühlung und Heizung, z.B. durch Strahlung, effiziente Pumpsysteme mit geringen Dimensionen erreichen.

Bisher wurden Drücke um $10^{4} \mathrm{~Pa}$ in einstufigen Systemen erreicht. Bild 19 zeigt den Druckverlauf der Diffusionspumpe von Bild 15.

\section{Zusammenfassung und Ausblick}

Zusammenfassend zeigen die hier vorgestellten Lösungen, dass die Mikrosystemtechnik sich hervorragend eignet, auch sehr komplexe physikalische Messsyteme zu miniaturisieren, ohne an Messempfindlichkeit und Zuverlässigkeit zu verlieren. Damit ist nicht nur ein Teil der gegenwärtig von großen Systemen bedienen, sondern es lassen sich auch viele neue Anwendungsgebiete erschließen. Auch die Komponenten und Subsysteme der Peripherie wie Flüssigkeitsverdampfer, Ventile, Druckmesssysteme und Druckstufen für die Probenzugabe sowie Mikro-Vakuumpumpen mit integrierter Druckmessung zu Erzeugung und Regelung der erforderlichen Unterdrucke können bezüglich Technologie, Dimension und Ressourcenbedarf mit diesen Mikrosystemen kompatibel sein.

Da die Herstellungsverfahren auf industriell verfügbaren Prozessen beruht, ist die industrielle Umsetzung dieser Systeme schon fortgeschritten. Während der $\mu \mathrm{GC}$ sich schon seit einigen Jahren als Produkt der SLS-Microtechnology, Hamburg, auf dem Markt ist, werden für die anderen beschriebenen Systeme kurzfristig entsprechende Produktmuster von kooperierenden Unternehmen vorgestellt werden.

\section{Danksagung}

Die vorgestellten Arbeiten wurden über viele Jahre von dem Land Hamburg, der Deutschen Forschungsgemeinschaft, dem BMBF, der EU sowie mehreren Industrieunternehmen finanziell unterstützt. 


\section{Literatur}

D.Sander, J. Müller, Selffocussing phase transmission grating for an integrated optical micro spectrometer, Sensors \&Actuators A 88 (2001),1-9

Uwe Lehmann, J. Müller, Analyse in miniature, Vacuum Solutions, Nov./Dez. 1998, S. 13-15

U. Lehmann, H. Plehn, O. Krusemark, J. Müller, A miniaturized gas chromatograph for autonomous and longtime measurements, Sensor 1999, B2.5, S. 155-158

www.sls-microtechnology.com

S. Zimmermann, S. Wischhusen, J. Müller: Micro flame ionization detector and micro flame spectrometer, Sensor \& Actuators B 63 (2000) 159-166

S.Zimmermann, P.Krippner, A.Vogel, J.Müller, Miniaturized flame ionisation detector for gas chromatography, Sens.”Actuat. B83, 2002, S. 285-289

S.Zimmermann, S. Wischhusen, J. Müller: Micro flame ionization detector and micro flame spectrometer, Sensor \& Actuators B 63 (2000) 159-166

J.-P. Hauschild, E. Wapelhorst, J. Müller, Mass spectra measured by a fully integrated MEMS mass spectrometer, Journal, International Journal of Mass Spectrometry, Vol. 264, Issue 1, 15 June 2007, Pages 53-60, DOI:10.1016/j.ijms.2007.03.014, Elsevier 2007, http://dx.doi.org/10.1016/j.ijms.2007.03.014

E. Wapelhorst, J.-P. Hauschild, J. Müller, Complex MEMS:A Fully Integrated TOF Micro Mass Spectrometer, Journal, Sensors and Actuators A 138 (physical), Vol. 138, Issue 1, 20 July 2007, Pages 22-27, doi:10.1016/j.sna.2007.04.041

http://dx.doi.org/10.1016/j.sna.2007.04.041

J.-P. Hauschild, E. Wapelhorst, J.Müller, The novel synchronous ion shield mass analyzer, J. Mass. Spectrom. 2009, 44, 1330-1337

M. Reinhardt, G. Quiring, R. M. Ramirez Wong, H. Wehrs, J. Müller, Helium detection using a planar integrated micro-mass spectrometer, International Journal of Mass Spectrometry 295 (2010), 145-148

Kuipers, W.J. and Müller, J., Sensitivity of a planar micro-flame ionization detector, Talanta 82 (2010) 1674-1679.

St. Vonderschmidt, J. Müller, A Novel Micro Paramagnetic Oxygen Sensor, MEMS 2010

M.Doms, A.Bekesch, J.Müller, A microfabricated Pirani Pressure Sensor operating near atmospheric pressure, J.Micromech.Microeng., 15/8 2005, 1504-1510 